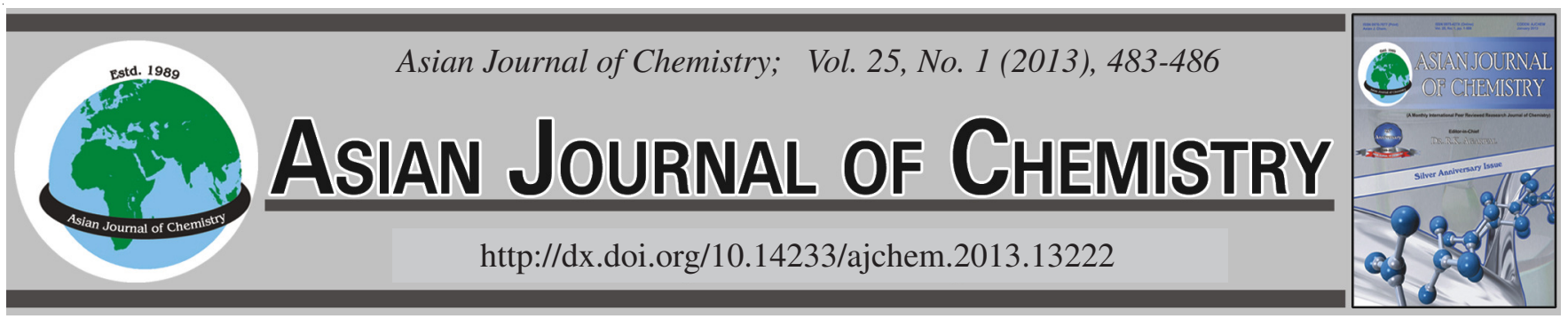

\title{
Synthesis of 1,4-Disubstituted 1,2,3-Triazoles via Three Component Coupling of Alcohol, Azide and Alkynes Using $\mathrm{CuO}$ Nanoparticles
}

\author{
Nagaraju Mittapelly ${ }^{1,2}$, Khagga Mukkanti ${ }^{2}$ and Buchi Reddy Reguri ${ }^{3, *}$
}

\begin{abstract}
${ }^{1}$ Inogent Laboratories Private Limited, A GVK BIO Company, Nacharam, Hyderabad-500 076, India
${ }^{2}$ Centre for Chemical Sciences and Technology, I.S.T, Jawaharlal Nehru Technological University, Kukatpally, Hyderabad-500 085, India

${ }^{3}$ Orchid Chemicals and Pharmaceuticals Ltd, Sholinganallur, Chennai-600 119, India
\end{abstract}

*Corresponding author: E-mail: nagunitrogen@yahoo.co.in

(Received: 10 December 2011;

Accepted: 1 August 2012)

AJC-11905

\begin{abstract}
A one-pot reaction for the synthesis of 1,4-disubstituted 1,2,3-triazoles from the alcohols via copper oxide nanoparticles (nano $\mathrm{CuO}$ ) catalyzed nucleophilic substitution of alcohol with azide, followed by azide-alkyne 1,3-dipolar cycloaddition (click reaction) is reported. 1,4-Disubstituted 1,2,3-triazoles are obtained in good to excellent yields from a variety of readily available alcohols without the need for the preactivation and isolation of the azide intermediates.
\end{abstract}

Key Words: 1,4-Disubstituted 1,2,3-triazoles, Azide, 1,3-Dipolar cycloaddition, $\mathrm{CuO}$ nanoparticles.

\section{INTRODUCTION}

The ever increasing demand for the novel medicinally active compounds and the laborious process of lead discovery and optimization have resulted in the continuous search for simple and efficient methods for generation of libraries for biological screening. Click chemistry has emerged as a fast and efficient approach to synthesize novel compounds with desired function making use of selected "near perfect" reactions ${ }^{1}$. The Huisgen 1,3-dipolar cycloaddition ${ }^{2}$ of azides and alkynes resulting in 1,2,3-triazoles is one of the most powerful click reactions.

Copper catalyzed ligation of organic azides and terminal alkynes has enjoyed much use since its discovery. Exclusive regioselectivity, wide substrate scope, mild reaction conditions and very high yields ${ }^{3}$ have made it the method of choice for making permanent connections by means of 1,4-disubstituted 1,2,3-triazoles. Since then, this reaction has been used for the construction of a variety of multivalent structures such as sugar heterodimers, glycoconjugates ${ }^{4}$, calix-sugars ${ }^{5}$ and dendritic and polymeric materials ${ }^{6}$. Furthermore, the one-pot multistep reaction involving the Wittig olefination, the Knoevenagel condensation, the Diels-Alder cyclization and $\mathrm{Cu}(\mathrm{I})$ - catalyzed alkyne-azide coupling has been explored ${ }^{7}$. Gratifyingly, the same level of success has been found when compared to the traditional methodology. In another variation, microwaveassisted one-pot reaction has generated a variety of triazoles directly from activated aryl halides and sodium azide ${ }^{8}$. In a similar fashion, taking advantage of anomeric activation, rapid one-pot syntheses of triazole-linked glycoconjugates have also been developed from readily available unprotected saccharides or saccharide acetates ${ }^{9}$. Several members of the 1,2,3- triazole family have indeed shown interesting biological properties, such as antiallergic ${ }^{10}$, antibacterial ${ }^{11}$ and anti-HIV activity ${ }^{12}$. Additionally, 1,2,3-triazoles are found in herbicides, fungicides and dyes ${ }^{13}$.

Despite these advances, there is a need to broaden the scope of one-pot multistep reaction in combination with click chemistry. Although organic azides are generally safe compounds, those of low molecular weight can be unstable and, therefore, difficult to handle ${ }^{14}$. This is especially true for small molecules with several azide functionalities that would be of much interest for the generation of polyvalent structures. Thus, a methodology that avoids isolation of organic azides is desirable. Aliphatic azides can be readily prepared from the corresponding halides by nucleophilic displacement or, in cases of aryl and vinyl azides, by a $\mathrm{Cu}(\mathrm{I})$-catalyzed reaction (vide infra) with sodium azide.

The substitution is especially facile when activated halides, such as allylic, propargylic and benzylic, are used. In present study we have studied the reactivity of alcohols in such reactions, which generally requires preactivation of hydroxyl group to convert in to azides ${ }^{15}$. Herein, we report a one-pot synthesis of 1,4-disubstituted 1,2,3-triazoles via three component coupling of secondary alcohol, trimethylsilyl azide and terminal alkynes in the presence of a catalytic amount of a $\mathrm{CuO}$ nanoparticles (Scheme-I). 


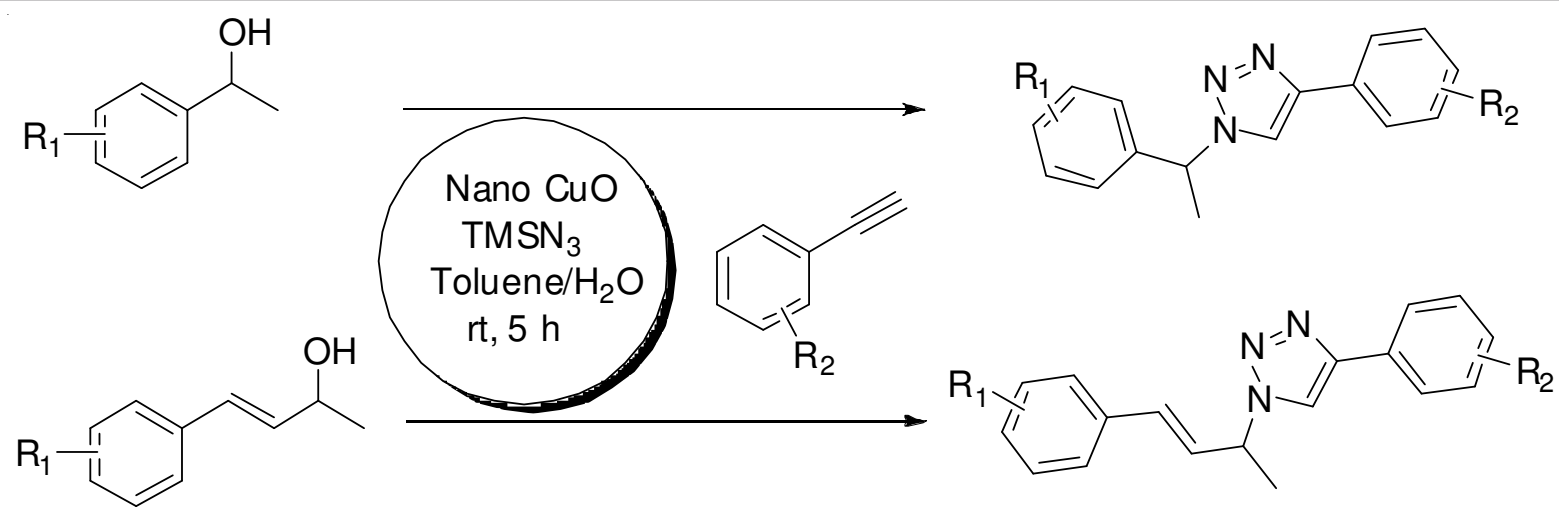

Scheme-I

\section{EXPERIMENTAL}

All chemicals were purchased from Sigma-Aldrich and S.D. Fine Chemicals, Pvt. Ltd. India and used as received. ACME silica gel (100-200 mesh) was used for column chromatography. All the other chemicals and solvents were obtained from commercial sources and purified using standard methods. The IR spectra of all compounds were recorded on a Perkin-Elmer, Spectrum GX FTIR spectrometer. The ${ }^{1} \mathrm{H},{ }^{13} \mathrm{C}$ NMR spectra were recorded on a Varian-400 MHz, BrukerAvance $300 \mathrm{MHz}$ spectrometer. Chemical shifts $(\delta)$ are reported in ppm, using TMS $(\delta=0)$ as an internal standard in $\mathrm{CDCl}_{3}$. ESI mass spectra were recorded on a Finnigan LCQ Advantagemax spectrometer.

General experimental procedure: A mixture of alcohol ( $1 \mathrm{mmol})$, trimethylsilyl azide $(2 \mathrm{mmol})$ and $\mathrm{CuO}$ nanoparticles $(3 \mathrm{~mol} \%)$ in toluene $(3 \mathrm{~mL})$ was stirred at $60^{\circ} \mathrm{C}$ for $3 \mathrm{~h}$. After complete consumption of the alcohol as indicated by TLC, alkyne $(1.2 \mathrm{mmol})$ and water $(2 \mathrm{~mL})$ was added and continued the reaction. After completion of the reaction, the reaction mixture was diluted with water and extracted with ethyl acetate $(2 \times 10 \mathrm{~mL})$. The combined organic layers were dried over anhydrous $\mathrm{Na}_{2} \mathrm{SO}_{4}$, concentrated in vacuo and purified by column chromatography on silica gel to afford the pure product. The product was well characterized by ${ }^{1} \mathrm{H} \mathrm{NMR},{ }^{13} \mathrm{C}$ NMR and mass spectroscopic analysis.

4-Phenyl-1-(1-phenyl-ethyl)-1H-(1,2,3)triazole (Table1, entry 1): ${ }^{1} \mathrm{H} \mathrm{NMR}\left(300 \mathrm{MHz}, \mathrm{CDCl}_{3}\right): \delta$ (ppm) 2.03 (d, $3 \mathrm{H}, J=7.554 \mathrm{~Hz}), 5.79-5.86(\mathrm{q}, 1 \mathrm{H}, J=7.6 \mathrm{~Hz}), 7.23-7.39$ $(\mathrm{m}, 8 \mathrm{H}), 7.55(\mathrm{~s}, 1 \mathrm{H}), 7.75(\mathrm{~d}, 2 \mathrm{H}, J=8.309 \mathrm{~Hz}) .{ }^{13} \mathrm{C} \mathrm{NMR}$ (75 MHz, $\mathrm{CDCl}_{3}$ ): $\delta$ (ppm) 21.23, 60.25, 118.20, 125.62, 126.52, 128.0, 128.56, 128.73, 128.99, 130.68, 139.90, 147.76 . ESI MS (m/z): $250(\mathrm{M}+\mathrm{H})^{+}$.

1-[1-(4-Bromo-phenyl)-ethyl]4-phenyl-1H-(1,2,3) triazole (Table-1, entry 2): ${ }^{1} \mathrm{H}$ NMR $\left(300 \mathrm{MHz}, \mathrm{CDCl}_{3}\right) \delta$ (ppm) $2.01(\mathrm{~d}, 3 \mathrm{H}, J=7.6 \mathrm{~Hz}) 5.75-5.82(\mathrm{q}, 1 \mathrm{H}, J=7.6 \mathrm{~Hz})$, 7.17 (d, 2H, $J=8.309 \mathrm{~Hz}), 7.27-7.39(\mathrm{~m}, 3 \mathrm{H}), 7.48(\mathrm{~d}, 2 \mathrm{H}$, $J=8.309 \mathrm{~Hz}) 7.56(\mathrm{~s}, 1 \mathrm{H}), 7.74(\mathrm{~d}, 2 \mathrm{H}) .{ }^{13} \mathrm{C} \mathrm{NMR}(75 \mathrm{MHz}$, $\left.\mathrm{CDCl}_{3}\right) \delta(\mathrm{ppm}) 21.24,59.70,118.21,120.19,125.71,127.98$, $128.18,128.65,128.77,132.26,139.80,147.75$. ESI MS $(\mathrm{m} / \mathrm{z}): 329(\mathrm{M}+2)^{+}$.

4-Phenyl-1-(1-p-tolyl-ethyl)- $1 H$-[1,2,3]triazole (Table1, entry 3): ${ }^{1} \mathrm{H}$ NMR $\left(300 \mathrm{MHz}, \mathrm{CDCl}_{3}\right) \delta(\mathrm{ppm}) 2.01(\mathrm{~d}, 3 \mathrm{H}$, $J=7.2 \mathrm{~Hz}$ ), 2.35 (s, 3H) 5.76-5.86 (q, 1H, $J=7.2 \mathrm{~Hz}$ ), 7.12 $(\mathrm{d}, 2 \mathrm{H}, J=7.9 \mathrm{~Hz}), 7.26-7.37(\mathrm{~m}, 5 \mathrm{H}), 7.5(\mathrm{~s}, 1 \mathrm{H}), 7.61(\mathrm{~d}$, $2 \mathrm{H}, J=7.9 \mathrm{~Hz}) .{ }^{13} \mathrm{C} \mathrm{NMR}\left(75 \mathrm{MHz}, \mathrm{CDCl}_{3}\right) \delta(\mathrm{ppm}) 21.26$, 21.34, 60.19, 117.97, 125.54, 126.59, 127.93, 128.52, 128.95, 129.43, 137.88, 139.92, 147.78. ESI MS (m/z): $264(\mathrm{M}+\mathrm{H})^{+}$.

4-Phenyl-1-(1-phenyl-propyl)-1H-[1,2,3]triazole (Table-1, entry 4): ${ }^{1} \mathrm{H}$ NMR $\left(300 \mathrm{MHz}, \mathrm{CDCl}_{3}\right) \delta$ (ppm) 0.98 $(\mathrm{t}, 3 \mathrm{H}, J=7.6 \mathrm{~Hz}), 2.27-2.42(\mathrm{~m}, 1 \mathrm{H}), 2.48-2.63(\mathrm{~m}, 1 \mathrm{H})$, $5.46(\mathrm{t}, 1 \mathrm{H}, J=6.798 \mathrm{~Hz}), 7.302-7.38(\mathrm{~m}, 8 \mathrm{H}), 7.59(\mathrm{~s}, 1 \mathrm{H})$, $7.75(\mathrm{~d}, 2 \mathrm{H}, J=7.6 \mathrm{~Hz}) .{ }^{13} \mathrm{C}$ NMR $\left(75 \mathrm{MHz}, \mathrm{CDCl}_{3}\right) \delta(\mathrm{ppm})$ $11.0,28.36,66.85,118.52,125.59,127.02,128.50,128.70$, 128.94, 130.68, 138.83, 147.64. ESI MS (m/z): $264.1(\mathrm{M}+\mathrm{H})^{+}$.

1-Benzhydryl-4-phenyl-1H-[1,2,3]triazole (Table-1, entry 5): ${ }^{1} \mathrm{H}$ NMR $\left(300 \mathrm{MHz}, \mathrm{CDCl}_{3}\right) \delta$ (ppm) $6.73(\mathrm{~s}, 1 \mathrm{H})$ 7.28-7.50 (m, 12H) $7.80(\mathrm{~d}, 2 \mathrm{H}), 7.95(\mathrm{~s}, 1 \mathrm{H}) .{ }^{13} \mathrm{C}$ NMR $(75$ $\left.\mathrm{MHz}, \mathrm{CDCl}_{3}\right) \delta$ (ppm) 68.09, 76.57, 77.00, 77.42, 119.57, $125.66,128.53,128.71,128.88,130.51,138.09,147.49$. ESI MS (m/z): $312.1(\mathrm{M}+\mathrm{H})^{+}$.

(E)-4-Phenyl-1-(4-phenylbut-3-en-2-yl)-1H-1,2,3triazole (Table-1, entry 6): ${ }^{1} \mathrm{H}$ NMR $\left(300 \mathrm{MHz}, \mathrm{CDCl}_{3}\right): \delta$ $1.85(\mathrm{~d}, 3 \mathrm{H}, J=6.8 \mathrm{~Hz}), 5.37-5.46(\mathrm{~m}, 1 \mathrm{H}), 6.40(\mathrm{dd}, 1 \mathrm{H}, J=$ $6.8 \mathrm{~Hz}, 15.8 \mathrm{~Hz}), 6.60(\mathrm{~d}, 1 \mathrm{H}, J=15.8 \mathrm{~Hz}), 7.24-7.40(\mathrm{~m}$, $8 \mathrm{H}), 7.74(\mathrm{~s}, 1 \mathrm{H}), 7.79(\mathrm{~d}, 2 \mathrm{H}, J=8.3 \mathrm{~Hz}) .{ }^{13} \mathrm{C}$ NMR $(75$ $\left.\mathrm{MHz}, \mathrm{CDCl}_{3}\right): \delta 20.7,58.4,117.8,125.6,127.6,127.9,128.3$, 128.6, 129.7, 130.6, 132.6, 135.5, 147.6. ESI MS (m/z): 276 $(\mathrm{M}+\mathrm{H}), 298(\mathrm{M}+\mathrm{Na})$.

(E)-1-(4-(4-bromophenyl)but-3-en-2-yl)-4-phenyl-1H1,2,3-triazole (Table-1, entry 7): ${ }^{1} \mathrm{H} \mathrm{NMR}\left(300 \mathrm{MHz}, \mathrm{CDCl}_{3}\right)$ : $\delta 1.86(\mathrm{~d}, 3 \mathrm{H}, J=6.8 \mathrm{~Hz}), 5.38-5.45(\mathrm{~m}, 1 \mathrm{H}), 6.40(\mathrm{dd}, 1 \mathrm{H}$, $J=6.5 \mathrm{~Hz}, 16.1 \mathrm{~Hz}), 6.51(\mathrm{~d}, 1 \mathrm{H}, J=16.1 \mathrm{~Hz}), 7.22-7.30(\mathrm{~m}$, $3 \mathrm{H}), 7.36-7.40(\mathrm{~m}, 2 \mathrm{H}), 7.44(\mathrm{~d}, 2 \mathrm{H}, J=8.5 \mathrm{~Hz}), 7.93(\mathrm{~s}, 1 \mathrm{H})$, $7.80(\mathrm{~d}, 2 \mathrm{H}, J=7.6 \mathrm{~Hz}) .{ }^{13} \mathrm{C} \mathrm{NMR}\left(75 \mathrm{MHz}, \mathrm{CDCl}_{3}\right): \delta 20.7$, 58.3, 117.8, 122.2, 125.6, 128.1, 128.5, 128.7, 130.5, 131.4, 131.7, 134.5, 147.7. ESI MS (m/z): $354(\mathrm{M})^{+}, 256(\mathrm{M}+2)$.

(E)-4-phenyl-1-(4-p-tolylbut-3-en-2-yl)-1H-1,2,3triazole (Table-1, entry 8): ${ }^{1} \mathrm{H}$ NMR $\left(300 \mathrm{MHz}, \mathrm{CDCl}_{3}\right): \delta$ $1.85(\mathrm{~d}, 3 \mathrm{H}, J=6.8 \mathrm{~Hz}), 2.35(\mathrm{~s}, 3 \mathrm{H}), 5.37-5.43(\mathrm{~m}, 1 \mathrm{H}), 6.34$ (dd, $1 \mathrm{H}, J=6.8 \mathrm{~Hz}, 15.6 \mathrm{~Hz}), 6.57(\mathrm{~d}, 1 \mathrm{H}, J=16.5 \mathrm{~Hz}), 7.09$ (d, 2H, $J=7.8 \mathrm{~Hz}), 7.23-7.29(\mathrm{~m}, 3 \mathrm{H}), 7.35-7.39$ (m, 2H), 7.74 (s, 1H), 7.79 (d, 2H, J = 7.8 Hz). ${ }^{13} \mathrm{C}$ NMR $(75 \mathrm{MHz}$, $\left.\mathrm{CDCl}_{3}\right): \delta 20.8,21.1,58.4,117.7,125.6,126.5,126.6,127.9$, $128.7,129.3,130.6,132.6,132.7,138.3,147.6$. ESI MS $(\mathrm{m} / \mathrm{z}): 290(\mathrm{M}+\mathrm{H})$.

(E)-1-(4-(4-Methoxyphenyl) but-3-en-2-yl)-4-phenyl$1 H$-1,2,3-triazole (Table-1, entry 9): ${ }^{1} \mathrm{H}$ NMR (300 MHz, 


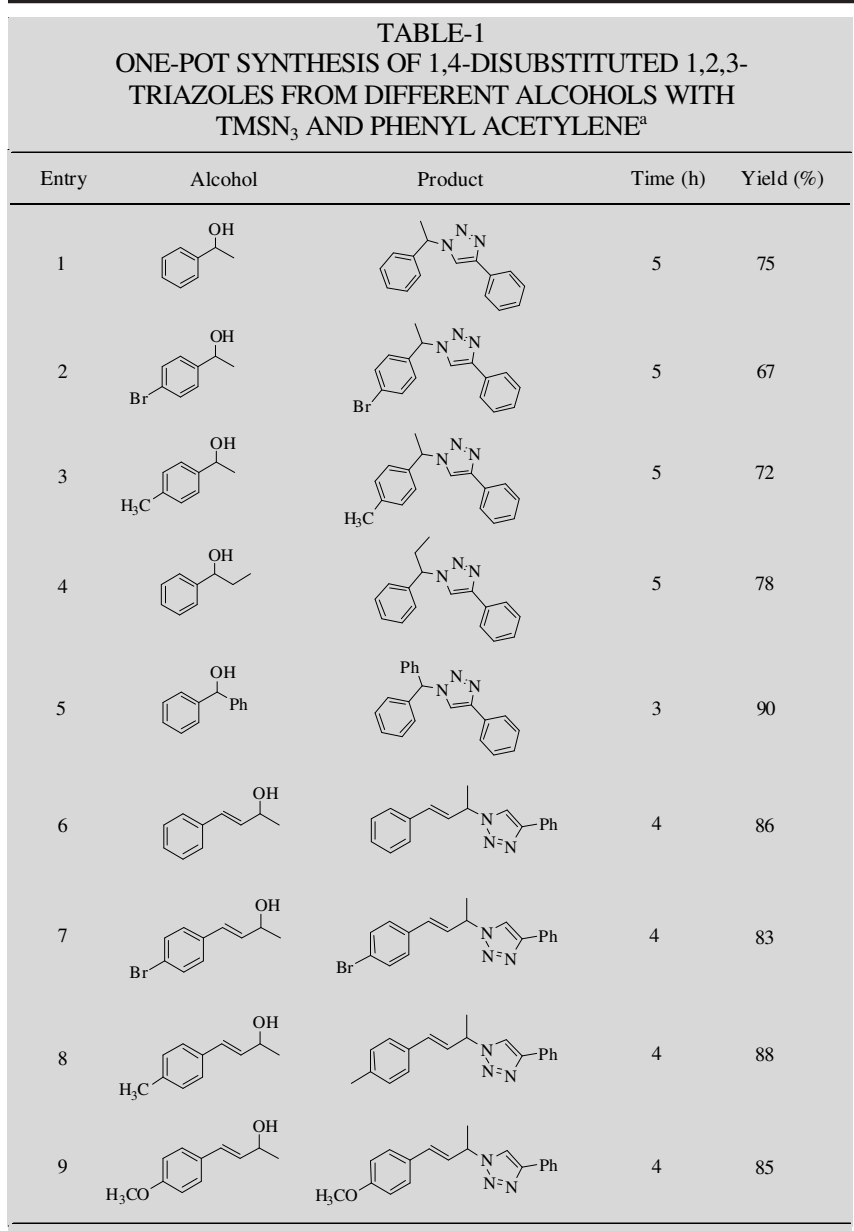

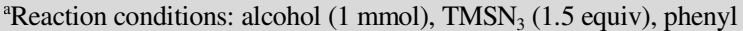
acetylene $(1.2 \mathrm{mmol})$ and toluene $(3 \mathrm{~mL})$, room temperature

$\left.\mathrm{CDCl}_{3}\right): \delta 1.83(\mathrm{~d}, 3 \mathrm{H}, J=6.8 \mathrm{~Hz}), 3.79(\mathrm{~s}, 3 \mathrm{H}), 5.35-5.40(\mathrm{~m}$, $1 \mathrm{H}), 6.24(\mathrm{dd}, 1 \mathrm{H}, J=6.8 \mathrm{~Hz}, 15.6 \mathrm{~Hz}), 6.57$ (d, 2H, $J=15.6$ $\mathrm{Hz}), 6.80$ (d, 2H, $J=8.7 \mathrm{~Hz}), 7.24-7.29(\mathrm{~m}, 3 \mathrm{H}), 7.34-7.37$ $(\mathrm{m}, 2 \mathrm{H}), 7.74(\mathrm{~s}, 1 \mathrm{H}), 7.78(\mathrm{~d}, 2 \mathrm{H}, J=7.8 \mathrm{~Hz}) .{ }^{13} \mathrm{C} \mathrm{NMR}(75$ $\left.\mathrm{MHz}, \mathrm{CDCl}_{3}\right): \delta 20.8,55.2,58.5,113.9,117.8,125.3,125.5$, $127.8,129.9,128.1,128.6,130.6,132.1,147.5,159.6$. ESI MS (m/z): $306(\mathrm{M}+\mathrm{H})$.

1-(1-Phenyl-ethyl)-4-p-tolyl-1H-[1,2,3]triazole (Table2, entry 1): ${ }^{1} \mathrm{H}$ NMR $\left(300 \mathrm{MHz}, \mathrm{CDCl}_{3}\right) \delta(\mathrm{ppm}) 2.01(\mathrm{~d}, 3 \mathrm{H}$, $J=7.2 \mathrm{~Hz}$ ), 2.35 (s, 3H) 5.76-5.86 (q, 1H $J=7.211 \mathrm{~Hz}), 7.12$ $(\mathrm{d}, 2 \mathrm{H}, J=7.9 \mathrm{~Hz}), 7.26-7.37(\mathrm{~m}, 5 \mathrm{H}), 7.50(\mathrm{~s}, 1 \mathrm{H}), 7.61(\mathrm{~d}$, $2 \mathrm{H}, J=7.9 \mathrm{~Hz}) .{ }^{13} \mathrm{C}$ NMR $\left(75 \mathrm{MHz}, \mathrm{CDCl}_{3}\right) \delta$ (ppm) 21.24, 21.30, 60.22, 118.0, 125.59, 126.56, 127.92, 128.53, 129.03, $129.43,137.87,140.02,147.85$. ESI MS (m/z): $264.1(\mathrm{M}+\mathrm{H})^{+}$.

4-(4-Methoxy - phenyl)-1 - (1-phenyl-ethyl)- 1 H [1,2,3]triazole (Table-2, entry 2): ${ }^{1} \mathrm{H} \mathrm{NMR}\left(300 \mathrm{MHz}, \mathrm{CDCl}_{3}\right)$ $\delta(\mathrm{ppm}) 2.02(\mathrm{~d}, 3 \mathrm{H}, J=7.2 \mathrm{~Hz}), 3.81(\mathrm{~s}, 3 \mathrm{H}), 5.77-5.84(\mathrm{q}$, $1 \mathrm{H}, J=7.2 \mathrm{~Hz}), 6.85(\mathrm{~d}, 2 \mathrm{H}, J=8.876 \mathrm{~Hz}), 7.27-7.36(\mathrm{~m}$, $5 \mathrm{H}), 7.46(\mathrm{~s}, 1 \mathrm{H}), 7.66(\mathrm{~d}, 2 \mathrm{H}, J=8.7 \mathrm{~Hz}) .{ }^{13} \mathrm{C}$ NMR $(75$ $\left.\mathrm{MHz}, \mathrm{CDCl}_{3}\right) \delta(\mathrm{ppm}) 21.29,55.30,60.19,114.19,117.50$, $126.53,126.97,128.50,129.02 .139 .7,159.67$. ESI MS (m/ $\mathrm{z}): 280.1(\mathrm{M}+\mathrm{H})^{+}$.

4-(4-Pentyl-phenyl) - 1 - (1 - phenyl-ethyl) - 1 H $[1,2,3]$ triazole (Table-2, entry 3): ${ }^{1} \mathrm{H}$ NMR $\left(300 \mathrm{MHz}, \mathrm{CDCl}_{3}\right)$ $\delta(\mathrm{ppm}) 0.85(\mathrm{t}, 3 \mathrm{H}, J=6.5 \mathrm{~Hz}), 1.24-1.33(\mathrm{~m}, 4 \mathrm{H}, J=3.6$ $\mathrm{Hz}), 1.53-1.68(\mathrm{~m}, 2 \mathrm{H}, J=7.9 \mathrm{~Hz}), 2.01(\mathrm{~d}, 3 \mathrm{H}, J=7.2 \mathrm{~Hz})$, $2.55(\mathrm{t}, 2 \mathrm{H}, J=7.9 \mathrm{~Hz}), 5.76-5.86(\mathrm{q}, 1 \mathrm{H}, J=7.2 \mathrm{~Hz}), 7.12$ (d,
$2 \mathrm{H}, J=7.9 \mathrm{~Hz}), 7.25-7.37(\mathrm{~m}, 5 \mathrm{H}) 7.5(\mathrm{~s}, 1 \mathrm{H}), 7.63(\mathrm{~d}, 2 \mathrm{H}$, $J=7.9 \mathrm{~Hz}) .{ }^{13} \mathrm{C} \mathrm{NMR}\left(75 \mathrm{MHz}, \mathrm{CDCl}_{3}\right) \delta(\mathrm{ppm}) 13.96,21.27$, 22.49, 30.98, 31.42, 35.67, 60.19, 118.00, 125.59, 126.06, $128.06,128.47,128.76,128.99,140.02,142.96,147.85 . \mathrm{ESI}$ MS (m/z): $320.2(\mathrm{M}+\mathrm{H})^{+}$.

3-[1-(1-Phenyl-ethyl)-1H-[1,2,3]triazol-4-yl]-pyridine (Table-2, entry 4): ${ }^{1} \mathrm{H}$ NMR $\left(300 \mathrm{MHz}, \mathrm{CDCl}_{3}\right) \delta$ (ppm) 2.01 $(\mathrm{d}, 3 \mathrm{H}, J=7.6 \mathrm{~Hz}), 5.75-5.79(\mathrm{q}, 1 \mathrm{H}, J=7.6 \mathrm{~Hz}), 7.28-7.34$ (m, 5H), $7.46(\mathrm{~d}, 1 \mathrm{H}, J=8.4 \mathrm{~Hz}), 7.57(\mathrm{~s}, 1 \mathrm{H}), 8.09(\mathrm{~d}, 1 \mathrm{H}$, $J=8.4 \mathrm{~Hz}), 8.75(\mathrm{~d}, 1 \mathrm{H} J=4.8 \mathrm{~Hz}), 8.92(\mathrm{~s}, 1 \mathrm{H}) .{ }^{13} \mathrm{C} \mathrm{NMR}$ $\left(75 \mathrm{MHz}, \mathrm{CDCl}_{3}\right) \delta(\mathrm{ppm}) 21.19,60.20,118.20,124.2,125.52$, $127.52,128.01,133.51,135.42,140.26,147.94,149.24$. ESI MS (m/z): $251.1(\mathrm{M}+\mathrm{H})^{+}$.

(E)-4-(3-Fluorophenyl)-1-(4-phenylbut-3-en-2-yl)-1H1,2,3-triazole (Table-2, entry 5): ${ }^{1} \mathrm{H}$ NMR $\left(300 \mathrm{MHz}, \mathrm{CDCl}_{3}\right)$ : $\delta 1.87(\mathrm{~d}, 3 \mathrm{H}, J=6.8 \mathrm{~Hz}), 5.38-5.48(\mathrm{~m}, 1 \mathrm{H}), 6.40(\mathrm{dd}, 1 \mathrm{H}$, $J=6.8 \mathrm{~Hz}, 16.1 \mathrm{~Hz}), 6.62(\mathrm{~d}, 1 \mathrm{H}, J=15.8 \mathrm{~Hz}), 6.91-7.01(\mathrm{~m}$, 1H), 7.27-7.39 (m, 6H), 7.50-7.59 (m, 1H), $7.75(\mathrm{~s}, 1 \mathrm{H}), 7.80$ $(\mathrm{d}, 1 \mathrm{H}, J=8.3 \mathrm{~Hz}) .{ }^{13} \mathrm{C} \mathrm{NMR}\left(75 \mathrm{MHz}, \mathrm{CDCl}_{3}\right): \delta 20.7,58.5$, 114.6, 114.9, 121.2, 125.5, 126.6, 127.4, 127.6, 128.4, 128.6, 130.2, 130.4, 132.8, 146.6, 161.4. ESI MS (m/z): $294(\mathrm{M}+\mathrm{H})$.

FROM DIFFERENT ALKYNES WITH TMSN AND ALCOHOLS

(E)-1-(4-Phenylbut-3-en-2-yl)-4-p-tolyl-1H-1,2,3triazole (Table-2, entry 6): ${ }^{1} \mathrm{H}$ NMR $\left(300 \mathrm{MHz}, \mathrm{CDCl}_{3}\right): \delta$ $1.85(\mathrm{~d}, 3 \mathrm{H}, J=6.6 \mathrm{~Hz}), 2.37$ (s, 3H), 5.38-5.45 (m, 1H), 6.40 (dd, $J=6.6 \mathrm{~Hz}, 16.1 \mathrm{~Hz}), 6.60(\mathrm{~d}, 1 \mathrm{H}, J=16.1 \mathrm{~Hz}), 7.17$ (d, $2 \mathrm{H}, J=8.1 \mathrm{~Hz}), 7.24-7.37(\mathrm{~m}, 5 \mathrm{H}), 7.68(\mathrm{~d}, 2 \mathrm{H}, J=8.1 \mathrm{~Hz})$, 
$7.70(\mathrm{~s}, 1 \mathrm{H}) .{ }^{13} \mathrm{C} \mathrm{NMR}\left(75 \mathrm{MHz}, \mathrm{CDCl}_{3}\right): \delta 20.7,21.2,58.3$, 117.4, 125.4, 126.5, 127.6, 127.8, 128.3, 128.6, 129.3, 132.6, 135.5, 137.7, 147.7. ESI MS (m/z): $290(\mathrm{M}+\mathrm{H})$.

(E)-2-(1-(4-Phenylbut-3-en-2-yl)-1H-1,2,3-triazol-4yl)pyridine (Table-2, entry 7): ${ }^{1} \mathrm{H} \mathrm{NMR}\left(300 \mathrm{MHz}, \mathrm{CDCl}_{3}\right)$ : $1.87(\mathrm{~d}, 3 \mathrm{H}, J=6.8 \mathrm{~Hz}), 5.41-5.50(\mathrm{~m}, 1 \mathrm{H}), 6.41(\mathrm{dd}, 1 \mathrm{H}, J=$ $6.8 \mathrm{~Hz}, 16.1 \mathrm{~Hz}), 6.63(\mathrm{~d}, 1 \mathrm{H}, J=16.1 \mathrm{~Hz}), 7.16-7.20(\mathrm{~m}$, 1H), 7.25-7.37 (m, 5H), 7.76 (ddd, $J=1.8 \mathrm{~Hz}, 7.7 \mathrm{~Hz}, 9.4$ $\mathrm{Hz}), 8.17-8.21$ (m, 2H), 8.51 (brd, $1 \mathrm{H}, J=4.9 \mathrm{~Hz}) .{ }^{13} \mathrm{C} \mathrm{NMR}$ $\left(75 \mathrm{MHz}, \mathrm{CDCl}_{3}\right): \delta 20.9,58.7,120.6,121.0,122.9,126.6$, 127.3, 128.4, 128.6, 133.1, 138.0, 148.1, 149.5. ESI MS $(\mathrm{m} / \mathrm{z}): 277(\mathrm{M}+\mathrm{H})$.

(E)-4-hexyl-1-(4-phenylbut-3-en-2-yl)-1H-1,2,3triazole (Table-2, entry 8): ${ }^{1} \mathrm{H}$ NMR $\left(300 \mathrm{MHz}, \mathrm{CDCl}_{3}\right)$ : 0.88 $(\mathrm{t}, 3 \mathrm{H}, J=6.5 \mathrm{~Hz}), 1.27-1.37(\mathrm{~m}, 6 \mathrm{H}), 1.59-1.70(\mathrm{~m}, 2 \mathrm{H})$, $1.79(\mathrm{~d}, 3 \mathrm{H}, J=6.9 \mathrm{~Hz}), 2.67(\mathrm{t}, 2 \mathrm{H}, J=7.9 \mathrm{~Hz}), 5.29-5.37$ $(\mathrm{m}, 1 \mathrm{H}), 6.34(\mathrm{dd}, 1 \mathrm{H}, J=6.6 \mathrm{~Hz}, 15.8 \mathrm{~Hz}), 6.53(\mathrm{~d}, 1 \mathrm{H}, J=$ 16.4 Hz), 7.24-7.37 (m, 6H). ${ }^{13} \mathrm{C}$ NMR (75 MHz, $\left.\mathrm{CDCl}_{3}\right): \delta$ 13.9, 20.7, 22.4, 25.7, 28.9, 29.4, 31.4, 50.1, 118.6, 126.5, 128.0, 128.2, 128.6, 132.3, 135.6, 148.4. ESI MS (m/z): 284 $(\mathrm{M}+\mathrm{H})$.

(E)-1-(1-(4-Phenylbut-3-en-2-yl)-1H-1,2,3-triazol-4yl)cyclohexanol (Table-2, entry 9): ${ }^{1} \mathrm{H}$ NMR (300 MHz, $\left.\mathrm{CDCl}_{3}\right)$ : 1.31-2.19 (m, 14H), 5.30-5.39 (m, 1H), $6.35(\mathrm{dd}, 1 \mathrm{H}$, $J=6.8 \mathrm{~Hz}, 15.8 \mathrm{~Hz}), 6.56(\mathrm{~d}, 1 \mathrm{H}, J=15.8 \mathrm{~Hz}), 7.23-7.43(\mathrm{~m}$, $6 \mathrm{H}) .{ }^{13} \mathrm{C}$ NMR $\left(75 \mathrm{MHz}, \mathrm{CDCl}_{3}\right): \delta 20.7,21.8,25.3,37.9$, 58.3, 69.4, 117.6, 126.5, 127.7, 128.3, 128.6, 132.6, 135.5, 155.5. ESI MS (m/z): $298(\mathrm{M}+\mathrm{H})$.

\section{RESULTS AND DISCUSSION}

Our study began with the direct conversion of an alcohol with trimethylsilyl azide into a alkyl azide intermediate which, without isolation, was treated with phenylacetylene to furnish the 1,3-dipolar cycloaddition product 1,4-disubstituted 1,2,3triazole in an one-pot operation using nano $\mathrm{CuO}$ as a catalyst. Water plays an important role in this reaction for (a) quenching of excess trimethylsilyl azide and (b) formation of copper acetylide from nano $\mathrm{CuO}$ and acetylene without any amine base. Table- 1 shows that the reaction proceeds smoothly to completion and the products were obtained in good yields. The reaction of various secondary benzylic alcohols bearing electron-rich and electron-poor substituents underwent the reaction smoothly and gave the products in good yield (Table1, entries 1-4). The reaction with benzhydrol and various allyl alcohols were even more faster and the products were formed in good to excellent yields (Table-1, entries 5-9).

In order to extend the scope of the reaction, several terminal alkynes were reacted with the azides of benzylic alcohol and allylic alcohol, which were generated in situ. It was observed that the reaction with a range of terminal alkynes, such as substituted phenyl acetylenes as well as aliphatic and hetero aromatic terminal alkynes, were equally effective and the resulting triazoles were obtained in good yields (85-65\%). Among the different terminal alkynes used in this present study, 4-methyl-, 4-methoxy-, 4-pentyl- and 3-fluoro-substituted phenyl acetylenes were found to be more reactive when compared to the heteroaromatic alkyne (Table-2, entries 1-7). In addition, aliphatic alkynes underwent the reaction smoothly to give the corresponding products in excellent yield under these optimized reaction conditions (Table-2, entries 8 and 9).

\section{Conclusion}

In conclusion, an efficient method for the synthesis of 1,4-disubstituted 1,2,3-triazoles from a variety of benzylic alcohol/allylic alcohol, trimethylsilyl azide and terminal alkynes has been developed via a simple one-pot, two-step procedure involving the azidation of alcohols, followed by 1,3-dipolar cycloaddition with terminal alkynes using nano $\mathrm{CuO}$ as the catalyst.

\section{REFERENCES}

1. H.C. Kolb, M.G. Finn and K.B. Sharpless, Angew. Chem. Int. Ed., 40, 2004 (2001).

2. R. Huisgen, in ed.: A. Padwa, In 1,3-Dipolar Cycloaddition Chemistry, Wiley: New York (1984).

3. V.V. Rostovtsev, L.G. Green, V.V. Fokin and K.B. Sharpless, Angew. Chem., Int. Ed., 41, 2596 (2002).

4. H. Srinivas and K. Sudhir, J. Org. Chem., 71, 364 (2006).

5. E.H. Ryu and Y. Zhao, Org. Lett., 7, 1035 (2005).

6. P. Wu, A.K. Feldman, A.K. Nugent, C.J. Hawker, A. Scheel, B. Voit, J. Pyun, J.M.J. Frechet, K.B. Sharpless and V.V. Fokin, Angew. Chem. Int. Ed., 43, 3928 (2004).

7. D.B. Ramachary and C.F. Barbas III, Chem. Eur. J., 10, 5323 (2004).

8. A. Prasad, W. Dehaen, V.V. Fokin and E.V. der Eycken, Org. Lett., 6, 4223 (2004).

9. S. Chittaboina, F. Xie and Q. Wang, Tetrahedron Lett., 46, 2331 (2005).

10. D.R. Buckle, C.J.M. Rockell, H. Smith and B.A. Spicer, J. Med. Chem., 29, 2269 (1986).

11. M.J. Genin, D.A. Allwine, D.J. Anderson, M.R. Barbachyn, D.E. Emmert, S.A. Garmon, D.R. Graber, K.C. Grega, J.B. Hester, D.K. Hutchinson, J. Morris, R.J. Reischer, C.W. Ford, G.E. Zurenko, J.C. Hamel, R.D. Schaadt, D. Stapert and B.H. Yagi, J. Med. Chem., 43, 953 (2000).

12. R. Alvarez, S. Velazquez, A. San-Felix, S. Aquaro, E. De Clercq, C.F. Perno, A. Karlsson, J. Balzarini and M.J. Camarasa, J. Med. Chem., 37, 4185 (1994).

13. H. Wamhoff, in eds.: A.R. Katritzky and C.W. Rees, In Comprehensive Heterocyclic Chemistry, Pergamon: Oxford, Vol. 5, pp. 669-732 (1984).

14. E.F.V. Scriven and K. Turnbull, Chem. Rev., 88, 351 (1988).

15. Y. Ju, D. Kumar and R. S. Varma, J. Org. Chem., 71, 6697 (2006). 